$\begin{array}{cl}\begin{array}{c}\text { Revue } \\ \text { de /histoire }\end{array} & \text { Revue de l'histoire des religions } \\ \text { des religions } & \begin{array}{l}\mathbf{3} \mathbf{2 0 0 6} \\ \text { Varia }\end{array}\end{array}$

\title{
Maîtriser le territoire alpin à l'heure des Lumières
}

Control the alpine territory in the Enlightenment: the diocese of Senj-Modruš in Habsburg's Croatia

Luc Oreskovic

\section{OpenEdition}

\section{Journals}

Édition électronique

URL : http://journals.openedition.org/rhr/5182

DOI : $10.4000 /$ rhr.5182

ISSN : 2105-2573

Éditeur

Armand Colin

\section{Édition imprimée}

Date de publication : 1 septembre 2006

Pagination : 319-344

ISBN : 2200-92105-5

ISSN : 0035-1423

\section{Référence électronique}

Luc Oreskovic, "Maîtriser le territoire alpin à l'heure des Lumières », Revue de l'histoire des religions [En ligne], 3 | 2006, mis en ligne le 27 janvier 2010, consulté le 20 avril 2019. URL : http:// journals.openedition.org/rhr/5182 ; DOI : 10.4000/rhr.5182 


\title{
Maîtriser le territoire alpin à l'heure des Lumières
}

\author{
Le diocèse de Senj-Modruš \\ en Croatie habsbourgeoise
}

Dans la chaîne des Alpes Dinariques, en Croatie habsbourgeoise, aux confins de l'empire ottoman et des territoires vénitiens, le diocèse catholique de Senj-Modruš renforce durant le XVIII siècle l'encadrement de ses administrés. Mais les déplacements sont malaisés dans un relief inhospitalier et les superstitions persistent. Par la diversité de son peuplement, cette région est aussi une frontière avec les "schismatiques", de confession orthodoxe, et avec l'islam ottoman. Dans le contexte de l'Aufklärung, une restructuration du réseau paroissial est réalisée par les évêques successifs qui soutiennent les missions organisées en montagne. D’un simple glacis militaire face aux Turcs, le diocèse devient une frontière intérieure où l'œuvre de conversion rencontre cependant un succès mitigé.

\section{Control the alpine territory in the Enlightenment: the diocese of Senj-Modruš in Habsburg's Croatia}

In the chain of Dinarics Alps, in Habsburgs Croatia, bordering the Ottoman empire and the Venetian territories, the catholic diocese of SenjModruš strengthens during XVIII ${ }^{\text {th }}$ century the frame of its managed. But travels are difficult in an inhospitable relief and superstitions persist. Because of the variety of its populating, this area is also a border with the "schismatic", of orthodox confession, and with the Ottoman Islam. In the context of Aufklärung, a reorganization of the parochial network is realized by the successive bishops who support the missions organized in mountains. Set like a simple military image in front of Turks, the diocese becomes an internal border where the work of conversion meets however a reserved success. 
Au XVIII ${ }^{\mathrm{e}}$ siècle, les contraintes inhérentes à l'espace géographique des Alpes Dinariques, dont les sommets culminent au-delà de 1700 mètres dans le territoire du diocèse catholique de SenjModruš, marquent le développement du réseau des paroisses et affectent la vie religieuse des populations dispersées dans les vallées éloignées des centres urbains du littoral dalmate. Situé dans le prolongement de l'arc alpin, à la confluence de l'Europe centrale et de la Méditerranée, le diocèse s'étend principalement à deux chaînes de montagnes, nommées Kapela et Velebit. Les obstacles naturels semblent conforter les diversités confessionnelles. Dès les pré-Lumières, les efforts déployés par le clergé invitent à une pratique religieuse plus maîtrisée : la maison d'Autriche soutient la rénovation de cette région de confins, au seuil de la Bosnie ottomane.

\section{LES DÉFIS DU RELIEF ALPIN}

Les difficultés rencontrées par l'Église catholique dans l'organisation du diocèse tiennent aux spécificités du paysage. Barrière naturelle qui sert de frontière avec les Turcs, la montagne est également le conservatoire des traditions mythologiques et de certaines formes du paganisme. Ces caractéristiques du milieu constituent autant de défis pour le clergé en charge de vastes territoires que la rigueur climatique rend parfois inhospitaliers.

\section{Les obstacles naturels}

En un lieu où les offensives turques menaçaient, le repli de la population catholique a délaissé d'importantes zones montagneuses. Lors du reflux ottoman, maîtriser le territoire consiste à restaurer les lieux de culte livrés par abandon à la lente érosion de la nature. Ainsi, au XVIII ${ }^{\mathrm{e}}$ siècle, l'église abbatiale Sainte-Croix dans la vallée encaissée de la Senjska Draga en amont de Senj, envahie par la végétation, fut patiemment restaurée par son abbé pendant de longues années ${ }^{1}$.

1. Voir K. Stošić, « Antun Jos. Cerovac i njegove propovijedi », Bogoslovska smotra, Zagreb, 1929, p. 186-193. Extrait du discours funèbre par le chanoine 
L'élan victorieux des Impériaux a permis de libérer en 1689 de nouvelles étendues montagneuses et les poljés adjacents. Un texte emphatique rédigé vers $1690^{2}$, peut-être par le capucin Marin Senjanin (de Senj), décrit les comtés de Lika et de Krbava : le cadre est verdoyant, accueillant, les terres sont fertiles. Un pays d'abondance s'offre aux catholiques appelés à s'y implanter. Leur retour dans ces terres après la longue occupation turque est le gain du vainqueur sur l'islam. Cette vision positive est tempérée, un an plus tard, par l'évêque Sébastien Glavinić qui décrit l'ensemble de son diocèse comme stérile et montagneux ${ }^{3}$. La pauvreté des habitants de l'intérieur des terres, notamment des paysans-soldats (Grenzer) enrégimentés par l'administration militaire des Confins, est constante. Même l'empereur et roi Joseph II, qui visite en mai 1775 cette partie déshéritée de la Croatie, est sensible aux insuffisances de la société. Le souverain note le 9 mai 1775 : «Depuis Raduč, nous chevauchons, et après Gospić, nous voyons quatre compagnies de Licaniens. Le pays semble être fertile et avoir de meilleures terres, mais le sol étant rougeâtre et cependant mélangé avec des pierres, il pousse peu de chose au-dessus, et les sols doivent être laissés reposés et déserts pendant quatre ou cinq ans, jusqu'à ce qu'on puisse les cultiver à nouveau. La région entière est exactement au plus haut degré de la pauvreté et de misère, au point que tous les villages mendient, se déchirent pour une mauvaise nourriture qui paraît misérable ${ }^{4}$. »

Cerovac en date du 17 septembre 1733 en l'honneur de Juraj Homolić, abbé de Sainte-Croix : «Elle avait été abandonnée pendant 200 ans et la forêt et la végétation l'avaient déjà envahi. Il s'est employé à lui donner l'éclat d'aujourd'hui. »

2. Brevis et compediosa duorum comitatuum Regni Croatiae Likae et Corbaviae descriptio, document manuscrit daté de 1695 conservé à l'Académie croate des sciences et des arts de Zagreb (HAZU, manuscrit XIX-33) et dans les archives diocésaines de Senj (Fasc. I br. 14). Un extrait a été traduit en croate par Mgr Mile Bogović et publié dans Croatica Christiana Periodica XV, 27, 1991, p. 117-118. La majeure partie est restituée en croate dans son ouvrage Hrvatsko glagoljsko tisućljeće (Millénaire glagolitique croate), Senjsko muzejsko društvo - Gradski muzej Senj, Senjski zbornik, 25e année, 1998, p. 104 et ss.

3. Archives de la Congrégation de la Propaganda Fide (ACPF), Acta 1692, vol. 62, f 67 / §22 - Croatia et Hongaria.

4. Ivan Erceg, « Dnevnik Josipa II. o prilikama u Hrvatskoj i na jadranskoj obali god. 1775. », Starine, 53, 1966, p. 247, 9 mai 1775. 
Le relief complique singulièrement l'administration du diocèse, longtemps dépourvu de voies de communication carrossables. En 1684, la très vaste étendue des paroisses du diocèse de Senj ne permet pas d'accomplir dans les meilleures conditions la charge pastorale des âmes. Senj, Bakar, Sv. Juraj sont d'anciennes paroisses côtières dont le territoire s'étend en profondeur aux vallées encaissées et aux hameaux dispersés dans les chaînes de montagne du Velebit. Avant 1689, les paroisses de l'arrière-pays, comme Brinje et Otočac, sont très proches des positions turques. Elles couvrent un relief de poljés aux abords de la chaîne intérieure des monts de Mala Kapela. Le développement du réseau des paroisses s'amorce au début du XVIII ${ }^{\mathrm{e}}$ siècle. Des zones isolées, dans l'arrièrepays montagneux, sont maintenant desservies plus fréquemment par les clercs séculiers. L'évolution est caractéristique dans la région de Krasno. Depuis le XVII ${ }^{\mathrm{e}}$ siècle, des populations ont traversé la montagne pour gagner les terres plus fertiles de Krasno et Devčić, s'installant auprès des anciennes routes, dans les poljés assez fertiles et dans les clairières, à Senjsko Bilo, et donnant naissance à des hameaux du nom des nouveaux arrivés, Vukelić par exemple, où le curé de Sveti Juraj, Ivan Vukelić, a décidé de rénover l'ancienne église de Sainte-Marie, ce qui fut fait en $1716^{5}$. À la place des anciens logements de montagne, on commence au début du XVIII siècle à construire des maisons solides avec des annexes pour le bétail. Puis de nouvelles églises et des succursales sont établies progressivement dans l'intérieur des terres. Cet essaimage existe aussi dans les marges du diocèse. Ainsi la chapelle Saint-Luc est à 5 heures de Karlobag ${ }^{6}$. L'église succursale Saint-Pierre de Bruvno est située à une distance de plus de deux heures de l'église mère de Gračac, ce qui rend pénibles les déplacements de la population et des prêtres, surtout l'hiver?

5. Ante Glavičić, «Usmena predaja o gradnji crkve svete Marije u Krasnu (Zapis Josipa Nagya, župnika u Krasnu) », Senjski zbornik, 1991, p. 227-235, note 11.

6. Archives diocésaines de Senj, F I br. 28 B, année 1733. Visite du diocèse de Senj-Modruš.

7. Archives diocésaines de Senj, BAS, Protocollum commissionis mixtae seu conscriptio parochialium filiarumque ecclesiarum Archidiaconatus et districtus Liccano-Corbaviensis Dioecesis Modrussiensis sub jurisdictione inclyti Regiminis Licani existentium (1768), f ${ }^{\circ} 10 \mathrm{v}$. 
Zrmanja, autre succursale, est à 6 heures de marche de Gračac ${ }^{8}$. La piste traversant l'abrupte chaine des monts Velebit était-elle souvent empruntée par les capucins de Karlobag pour visiter le comté de Lika dont les âmes leur étaient confiées ? Afin de résider au cœur de cette région, deux capucins sont installés à partir de 1721 à Kaniža. De la même façon, deux pauliniens résident à Gvozd dans les montagnes. Mais le cloisonnement des territoires apparaît nettement en 1722 dans le refus de certaines collégiales de remettre à l'évêque les fruits de la dîme des « églises d'outre forêt » 9 .

Aux alentours de Senj, jusqu'à la fin du XVIII ${ }^{\mathrm{e}}$ siècle, les voyageurs devaient emprunter des pistes souvent tortueuses et escarpées. Même pour un bon cavalier ou un voyageur à dos de mulet, traverser la chaîne des Alpes Dinariques constituait une véritable épreuve. Aussi, les évêques successifs ont rarement résidé durablement à Senj, préférant Trsat, Bakar ou Novi, à l'exception de Juraj Vuk Tcholić (Čolić) natif de la ville. Cette localisation septentrionale de l'évêque négligeait délibérément le clergé séculier du sud du diocèse qui résidait au-delà des montagnes, en Lika et Krbava. L'éloignement des prélats reste un frein à la prompte application des consignes qu'ils édictent. La diffusion de la circulaire ${ }^{10}$ rédigée par l'évêque Benzoni en novembre 1740 pour enjoindre à son clergé de préparer le cérémonial funèbre en l'honneur du monarque Charles VI démontre la difficile maittrise des distances. La circulaire publiée le 30 novembre par l'évêque, à Trsat, est transmise à la chancellerie épiscopale de Senj le 2 décembre, puis de paroisse en paroisse selon un itinéraire qui permet de franchir les chaînes des monts Velebit en période hivernale dans les meilleurs délais. Mais la circulaire arrive seulement

8. Emanuel Hoško, « Biskup Pijo Manzador i preuređenje župa u Lici i Krbavi », Riječki teološki časopis, 3, 1995, p. 270.

9. Archives diocésaines de Senj, série BAS, F I br. 21 B ; 5 février 1722. L'évêque a tenté de redistribuer les revenus des différents chapitres comme l'atteste un document de l'année 1722. Le 5 février de cette année, les chapitres (ou collégiales) de Bribir, Grižane, Belgrad, Drivenik, Hreljin, Bakar et Grobnik indiquent à nouveau à l'évêque de Senj que les églises d'outre forêt n'appartiennent pas à l'évêché de Senj et qu'ils n'ont pas à donner le dixième au chapitre de Senj mais qu'ils dépendent de la juridiction du seul vicaire de Vinodol.

10. Archives diocésaines de Senj, F I br. 36 B. 
le 30 décembre à Jablanac. Jusqu'à la fin du siècle, la distance entre Senj et l'intérieur du diocèse reste un obstacle à la communication entre les acteurs de la vie religieuse. Ainsi, en 1767, les missionnaires jésuites notent que six heures de voyage à travers la montagne sont nécessaires pour rallier Otočac et son doyenné ${ }^{11}$. Si le nord du diocèse est relié à l'intérieur de la Croatie par la route Caroline, initiée par l'empereur Charles VI en 1726, sa partie centrale doit attendre pour que la route Joséphine soit terminée en 1779 sous Joseph II.

\section{Les montagnes, refuges du surnaturel}

Le domaine des croyances populaires est vaste. Mechthild Pečik a exploité dans plusieurs villages de Croatie intérieure et littorale ${ }^{12}$, parfois dans les territoires des anciens diocèses de Senj et de Krk, les témoignages individuels de nos contemporains se rapportant à l'attitude devant la mort ${ }^{13}$ : l'existence d'un rituel spécifique lors des funérailles des prêtres, le rythme codé de la sonnerie des cloches de l'église paroissiale en fonction de la condition sociale du défunt, l'évocation d'un surnaturel particulier ${ }^{14}$, sont quelques-uns des aspects qui dénotent la permanence de traditions populaires encore éloignées de la conformité tridentine. Superstitions et cultes païens subsistent dans les zones de montagnes. Dans le diocèse de Senj-Modruš, en Croatie, les denses forêts des monts Velebit et de Kapela sont réputées abriter les fées, notamment la fée du Velebit (vila Velebita), mais aussi les vukodlaci, (sg. vukodlak) variété de lycanthropes tenant à la fois du loup-garou et du vampire. L'identité culturelle locale se rapproche cependant d'un fond commun aux Alpes sud-orientales. Les croyances pré-chrétiennes étudiées dans le Frioul sont tout aussi vigoureuses dans les Alpes Dinariques et en l'occurrence dans le diocèse de Senj-Modruš. Carlo Ginzburg

11. Archivum Romanum Societatis Iesu ARSI), Austr. $222, f^{\circ} 21 \mathrm{v}$.

12. Notamment en Zagorje, Turopolje, Medjimurje, Prigorje, Primorje et sur l'île de Krk.

13. Mechthild Pečik, Der Tod im Dasein des Menschen. Der Tod und sein Umfeld untersucht und dargestellt am Beispiel Kroatien, Tübingen, 1984.

14. Pečik, voir par exemple p. 78 la référence aux vampires. 
admet le parallélisme des traditions entre Frioul et Dalmatie ${ }^{15}$. Les superstitions, la magie et la divination, considérées comme des phénomènes religieux lato sensu, restent particulièrement vivaces dans le Sud de l'Autriche, la Slovénie et la Croatie, régions où l'interdiction des persécutions pour sorcellerie n'intervient qu'à partir des années 1750 ${ }^{16}$. En Croatie, comme en Pologne, en Hongrie et dans les territoires autrichiens où le diable est une figure bien présente ${ }^{17}$, la première moitié $\mathrm{du} \mathrm{XVIII}^{\mathrm{e}}$ siècle demeure fertile en événements où son intervention est signalée. Les témoignages recueillis par Nevena Skrbić enrichissent notre connaissance des croyances populaires dans le diocèse de Senj-Modruš ${ }^{18}$.

Les ressources spirituelles d'une civilisation agraire ne se résument pas à l'invocation de saints protecteurs. Les vieux démons païens de la nature, l'animisme et la fonction protectrice à l'intérieur d'une communauté sont cumulés. Les kresnik (bonnets de chance) évoluent dans un monde onirique et engagent le succès des récoltes lors de combats nocturnes. Ces hommes s'apparentent aux benandanti signalés dans le Frioul voisin entre 1580 et 1650 par les procès de l'Inquisition ${ }^{19}$. En Slovénie, le kresnik local, perçu comme un esprit bienveillant, combat, avec ceux des territoires voisins, pour que le territoire des vainqueurs devienne celui des riches récoltes, tandis que le domaine des perdants subira la grêle et les mauvaises récoltes. Dagmar Burkhart établit un lien entre les démons de la nature en Slovénie et ceux du Nord de la Dalmatie, à savoir les krsnik adversaires des vampires selon les croyances populaires des habitants du Nord de la Dalmatie et sur la côte de l'Istrie ${ }^{20}$. Maja Bošković-Stulli

15. Carlo Ginzburg, Les batailles nocturnes, Paris, 1980, p. 212-213.

16. Gábor Klaniczay, «Bûchers tardifs en Europe centrale et orientale », in Robert Muchembled (dir.), Magie et sorcellerie en Europe du Moyen-Âge à nos jours, Paris, 1994, p. 221 et 223.

17. Robert Muchembled, Une histoire du diable, XII $-X X^{e}$ siècles, Paris, 2000, p. 216.

18. Nevena Škrbić, «Pojedini elementi duhovne kulture primorskih Bunjevaca », Senjski zbornik, 27, 2000, p. 217-226.

19. Jean Delumeau, Le catholicisme entre Luther et Voltaire, Paris, 1979, p. 248.

20. Dagmar Burkhart, «Kulturraum Balkan - Studien zur Volkskunde und Literatur Südosteuropas », in Lebensformen, Band 5, Berlin, Hamburg, 1989, p. 80. 
donne une origine commune à ces krsnik, sorciers et sorcières, et les relie aux ancêtres sorciers et esprits protecteurs du clan et de la parenté. Sous l'influence de la chrétienté, les krsnik sont opposés aux sorciers, sorcières et vampires ${ }^{21}$, et sont ressentis comme complémentaires de la religion. La sorcellerie reprend cette dualité, notamment dans l'île de Rab, diocèse insulaire voisin du diocèse de Senj-Modruš, comme en attestent les documents analysés par Carlo Ginzburg pour l'année 1661. Des sorcières décrivent les dommages qu'elles ont infligés aux champs de blé et aux vignes à l'instigation du démon ${ }^{22}$. Outre la réminiscence d'un culte agraire, ce récit est une évocation de la menace diabolique qui pèse, comme un anti-cérémonial, sur la source des éléments du sacrifice de la messe, le pain et le vin.

Le lien entre nature et surnature est indicible. En témoigne la dévotion à sainte Lucie qui se superpose ainsi au culte de la fertilité. La jeune vierge martyre, associée dans la tradition à la lumière (lux), et dont la fête fixée au 13 décembre correspondait à la date du solstice d'hiver et à la journée la plus courte, était encore associée en Croatie à des rituels agraires ou même à des pratiques divinatoires ${ }^{23}$. À l'est des monts Velebit, en Lika, la Lucija est davantage celle des ténèbres, comme une figure de terreur. La veille du jour de fête, à la lueur des bougies et revêtus d'un drap de lin surmonté d'une citrouille percée de trous figurant les yeux, le nez et la bouche, les garçons de Lika se déplacent la nuit dans les villages, s'amusant à faire peur sous les fenêtres des maisons ${ }^{24}$. Jour de la bénédiction des gerbes de céréales en Europe centrale, cette date est marquée en Croatie, par exemple en Lika, par le geste d'ensemencer de quelques grains de blé ou d'orge un récipient rempli de la terre la plus noire. Chaque

21. Burkhart, p. 82.

22. Ginzburg, p. 212.

23. Michel Revelard, Guergana Kostadinova, Le livre des masques Masques et costumes dans les fêtes et carnavals traditionnels en Europe, Tournai, 1998, p. 23.

24. Leopold Kretzenbacher, Sancta Lucia und die Lutzelfrau, Volksglaube und Hochreligion im Spannungsfeld Mittel- und Südosteuropas, Südosteuropaïsche Arbeiten 53, München, 1959, p. 49. 
foyer regarde croître ce semis ; à Noël une bougie est plantée au milieu des brins de blé25 qui verdissent jusqu'à la fête de l'Épiphanie quand ils sont donnés en pâture aux agneaux et plus spécialement à la brebis qui vient de donner la vie. Le cycle végétal rejoint le cycle de la chair dans une évocation pastorale et christique.

\section{LA MONTAGNE, FRONTIÈRE CONFESSIONNELLE}

$\mathrm{Au}$ relief compartimenté semble répondre la mosaïque des peuplements qui se juxtaposent au gré des migrations sur le territoire du diocèse. Les musulmans autochtones n'ayant pas suivi le retrait ottoman en 1685-1689 se convertissent au catholicisme. Déjà implantés dans certains secteurs du diocèse durant le XVII siècle, les «Valaques schismatiques », de confession orthodoxe, voient leur nombre augmenter dans les territoires reconquis que les Croates catholiques ne peuvent coloniser à eux seuls. Dans les vallées plus ou moins enclavées, dans les poljés isolés et sur les plateaux karstiques, le catholicisme est parfois fragilisé. Un cloisonnement des communautés confessionnelles s'esquisse.

\section{Une frontière avec les « schismatiques »}

Dans certaines régions, le relief montagneux devient un enjeu de prééminence confessionnelle. En ce sens, l'édicule, la croix, la chapelle et l'église ont un rôle totémique. Une compétition entre les confessions marque le relief. Se pose la question de l'antériorité des édifices. Dans la localité de Vodoteč, une église orthodoxe dédiée à Saint Michel archange fut élevée sans qu'il ne subsiste de traces plus anciennes d'un quelconque bâtiment ${ }^{26}$. De la même façon, le naturaliste Balthasar Hacquet remarquait en 1804 que les orthodoxes multipliaient sur les montagnes en Carniole voisine les constructions

25. Yvonne De Sike, Fêtes et croyances populaires en Europe, au fil des saisons, Bordas, 1994, p. 43.

26. Zorislav Horvat, «Srednjovjekovna sakralna arhitektura u Brinju i okolici », Senjski zbornik, 27, 2000, p. 138. 
d'églises ${ }^{27}$. Joseph II avait même voulu fermer ces églises superflues. La motivation essentielle de ces constructions résiderait dans la quête d'offrandes que pratiquaient les orthodoxes ${ }^{28}$. Considérons également la délimitation d'un espace confessionnel spécifique.

En 1700, l'évêque Martin Brajković dresse un inventaire des positions «schismatiques » dans son diocèse ${ }^{29}$. Dans sa lettre au pape Clément XI, l'évêque mentionne la forte présence de « schismatiques » dans les territoires repris sur les Turcs ${ }^{30}$. L'ampleur du problème qui se pose à lui est exposée dans sa lettre à la Congrégation de la Propaganda Fide. S'il mentionne des bourgades dans la région au cœur des montagnes, du côté de la mer (mare versus), depuis longtemps habitées par des familles «schismatiques», comme Brlog, Sveti Juraj, Krmpote, Jablanac et Krasno, l'abondance des orthodoxes est signalée aux abords de Modruš (quae Schismatici infestatione veneni reperiantur immunes), d'Otočac et du poljé de Gacka où ils seraient plus d'un millier, et dans certains villages de la paroisse de Brinje, comme Dabar, Prokike et Luchane. Dans cette dernière paroisse, les « schismatiques » qui fréquentent souvent les églises catholiques sont inquiétés par leurs prêtres. Des « schismatiques » exercent leur rite dans Kosinj. Leur ministère ne manque dans aucune des localités de Czareno Polje, Muniava et Maravicza, dans le secteur de Oštarije et Ogulin où se trouvent 200 maisons de «schismatiques » ${ }^{31}$. Le déséquilibre entre les communautés, causé par l'évolution démographique et l'installation de nouveaux colons,

27. François Ruegg, À l'Est, rien de nouveau - De la barbarie à la civilisation ? - Les marches impériales et l'Europe, Genève, 1991, p. 45. Cite Balthasar Hacquet : «Il ne leur suffit pas d'avoir une église au village, mais dans plusieurs communes ils en possèdent de sept jusqu'à neuf qui se trouvent sur des montagnes éloignées de plusieurs milles et qui sont dédiées à différents saints. »

28. Ruegg.

29. Radoslav Lopašić [sakupio i uredio], Spomenici Hrvatske Krajine, Knjiga III, od godine 1693. do 1780. i u dodatku od g. 1531. do 1730., p. 189-193, document CIV.

30. Daniele Farlati, Illyrici Sacri..., tomus quartus, ecclesiae suffraganeae metropolis Spalatensis, auctore Daniele Farlato, presbytero Societies Jesu, Venetiis, apud Sebastianum Coleti, MDCCLXIX, p. 153 : ...et Schismaticorum, qui copiosi sunt, (...).

31. Farlati, p. 154. 
apparait nettement dans les éléments statistiques relatifs à chacune des capitaineries (Hauptmannschaften), étudiés par Karl Kaser pour la première moitié du XVIII ${ }^{\mathrm{e}}$ siècle $^{32}$. En 1712, à Korenica, il y avait seulement 27 catholiques pour 1786 Valaques et à Bunić seulement 18 catholiques pour 1488 Valaques $^{33}$. Le cas inverse des proportions se rencontre à Ostrovica où, pour 361 catholiques, on comptait seulement 57 Valaques. Mais, à cette époque, pour 8 villages entièrement catholiques, il en existe 17 qui sont entièrement orthodoxes. Des variations spectaculaires concernent Bilaj qui passe de 536 âmes à 1296 en 1746, soit une augmentation de $142 \%$; Bunić qui passe de 3326 âmes à 5922 en 1746, soit une augmentation de $78 \%$. Il est évident que les orthodoxes croissent plus vite en nombre que les catholiques.

Dans sa lettre en date du 12 septembre 1722 au conseil de la guerre de Graz, qui exerce le droit de patronage en plusieurs paroisses de la frontière militaire, le gardien du couvent des capucins de Karlobag, frère Damas de Fiume (Rijeka), exprime ses inquiétudes devant plusieurs formes de prosélytisme exercées par les «schismatiques » en Lika et en Krbava parmi les catholiques ${ }^{34}$. Le clergé catholique coexiste parfois péniblement auprès de la forte minorité orthodoxe. L'évêque Benzoni note en 1741 que, là où les catholiques vivent mélangés avec les «schismatiques », la moralité de la population est assez faible. Après avoir nommé l'évêque uniate Théophile Pašić comme son vicaire chargé des fidèles de «confession grecque », l'évêque Benzoni se prend à espérer que la situation va évoluer favorablement ${ }^{35}$. Pourtant, en 1742, un clerc donne un témoignage

32. Karl Kaser, Freier Bauer und Soldat. Die Militarisierung der agrarischen Gesellschaft an der kroatisch-slawonischen Militärgrenze (1535-1881), Wien, Köln, Weimar, 1997, p. 249 ; Tabelle 32 : Das Bevölkerungswachstum im Likaner Distrikt 1712 bis 1746.

33. Kaser, p. 248.

34. Archives militaires de Vienne (Kriegsarchiv) - Innerösterreichischer Hofkriegsrat : série Croatica, fo 5 r-v. : Lettre en date du 12 septembre 1722 signée du frère Damasus Fluminensis, gardien du couvent des capucins de Karlobag et adressée ( $f^{\circ} 10 v$.) au président du conseil de la guerre d'Autriche intérieure à Graz. 1741.

35. Rapport de la visite ad limina de l'évêque Jean Antoine de Benzoni en 
négatif sur les mœurs des habitants qu'il côtoie. Affecté à Lovinac, « dans les limites de la Lika », le curé Anton Pavletić dit se trouver «au milieu de gens barbares, suant du sang pour les âmes ». Il remarque que « les gens d'ici sont grossiers, ignorants » et se situent vraiment entre les mœurs des « schismatiques » et des Turcs. Dénonçant la multitude des « homicides, usures, pillages (ou rapts), vols, brigandages et autres crimes et forfaits répandus ${ }^{36} »$, il réprimande la population en la menaçant d'une mort sans repos. C'est souvent au péril de sa vie qu'il prêche la parole de Dieu et l'Évangile chaque jour de fête. Repoussé, inquiété, le curé professe pourtant à la Propaganda Fide sa volonté de montrer «dans les vignes du Seigneur toutes les activités et de s'approcher en même temps du salut jusqu'à la dernière heure de sa vie ${ }^{37}$ ». En 1763, le clerc séculier Giovanni Hreglianović ne souhaite pas obtenir la cure de Ribnik, alors vacante mais d' «air malsain ${ }^{38} »$. L'existence d'une forte minorité orthodoxe est sans doute pour beaucoup dans cette allusion au climat détestable de la localité. À la même époque, à Bruvno, dans le sud du diocèse, les catholiques, assez peu nombreux, avaient tendance à quitter une contrée où ils étaient dispersés parmi une population orthodoxe, dans un secteur frontalier de la Bosnie ottomane et des musulmans. Un rapport de 1768 souligne cette situation difficile : Situs est cappellantus in extremo confinio versus Bosniam Turcicam inter perversissimos Schismaticis per quorum temeritatem ac militiam, ne Catholici vel pervertantur vel ad emigrationem cogantur ${ }^{39}$.

36. Archives de la Sacrée Congrégation de la Propaganda Fide (SCPF), série S.C., Dalmazia 9 (1741-1745), f $\mathrm{f}^{\circ}$ 283r : Gens enim haec inter Schismaticos et Turcas rituata, iddiomatis Illyrici, continuis : praescinto a non dicendis : homicidÿs, usuris, rapinis, furtis, latrocinis, alï̈aq-, scelertissimis publicis facinoribus infecta jacet.

37. SCPF, série S.C., Dalmazia 9 (1741-1745), f ${ }^{\circ}$ 283r-284r.

38. SCPF, Série «Scritture riferite nei congressi » (SC) : Dalmazia 12, $f^{\circ} 315 v$. Lettre de Giovanni Hreglianovic en date de Senj le 7 août 1763.

39. Archives diocésaines de Senj, BAS, Protocollum commission mixtae seu conscriptio parochialium filiarumque ecclesiarum Archidiaconatus et districtus Liccano-Corbaviensis Dioecesis Modrussiensis sub jurisdictione inclyti Regiminis Licani existentium (1768), f ${ }^{\circ} 10 \mathrm{v}$. 


\section{Les Turcs et l'islam derrière la montagne}

À l'extrémité des Alpes, en Croatie, l'islam a déjà mordu sur le territoire montagneux depuis la fin du $\mathrm{XV}^{\mathrm{e}}$ siècle. La résistance opposée par les chrétiens s'appuie sur un milieu naturellement fortifié par les escarpements rocheux. Limitrophe de la Bosnie ottomane, le diocèse de Senj-Modruš reste une sentinelle face à l'islam aux $\mathrm{XVII}^{\mathrm{e}}$ et $\mathrm{XVIII}^{\mathrm{e}}$ siècles. Parfois pénétrées profondément par les raids militaires ottomans, les montagnes constituent une barrière âprement défendue, où le danger est permanent. Réciproquement, les chrétiens traversent ces montagnes pour attaquer les positions turques, comme le rapporte l'érudit segnan Pavao Ritter Vitezović. Ainsi, dans sa chronique pour l'année 1679, il précise : «Trois cents Segnans arrivèrent en Lika à côté de Novi [Lički Novi, près de Gospić], à travers le Velebit, et prirent beaucoup de butin sur l'ennemi, mais ne se retirèrent pas, leurs cavaliers se dispersant dans les villages et vers les tours turques, les fantassins allant danser leur ronde et les autres s'endormant, quand soudainement surgirent les Turcs. Les nôtres se retirèrent dans le désordre et le butin retourna dans les mains des Turcs. Les Segnans laissèrent 21 morts et 8 furent emprisonnés par les Turcs. Les Turcs laissèrent 30 morts et 7 prisonniers ramenés à Senj ${ }^{40}$. L'angoisse de rencontrer le Turc est donc bien présente. Le chant épique des uskoks de Senj, intitulé « Les jeunes filles de Senj partent de bonne heure ${ }^{41} »$, relate la mésaventure survenue à la jeune fille de la ville, Klara Daničić, qui allait chercher de l'eau à une source claire de Vratnik, au pied du col ouvrant la chaîne des montagnes sur un arrière-pays réputé hostile et sillonné par l'envahisseur turc. Elle échappe de peu au séducteur turc Harambaša. Cette chanson, dont la prose fut modifiée au cours des années mais qui conserve l'essentiel du récit d'origine, peut être datée du XVII ${ }^{\mathrm{e}}$ siècle. Elle célèbre la lutte contre l'infidèle et dénonce la pratique du rapt. Moins menaçant après 1689, l'islam est pourtant

40. Vjekoslav Klaić, Život i djela Pavla Ritter Vitezovića (1652-1713), Zagreb 1914, p. 33.

41. Voir A. Glavičić, «Rano rane senjkinje divojke, stara uskočka pjesma iz Senja », Senjski zbornik, 24, 1997, p. 253-266. 
encore proche géographiquement, implanté en Bosnie ottomane. Est-il attirant pour certains des chrétiens peu attachés à leur foi ? Un épisode relaté par l'ingénieur militaire Marsigli en 1700 montre comment les auxiliaires d'un marchand de Senj se firent «Turcs» lors de leur déplacement à Banja Luka en Bosnie, pour s'approprier un capital ${ }^{42}$. Signe des temps et expression d'un art populaire du XVIII ${ }^{\mathrm{e}}$ siècle, le détail d'un retable de l'église de Kosinj, au cœur des monts Velebit, représente le cavalier saint Georges écraser un monstre anthropomorphe dont la tête humaine et barbue ressemble à celle d'un Turc ${ }^{43}$. L'analogie avec l'épisode légendaire de l'aide apportée aux Croisés pour mettre en fuite les Sarrasins ${ }^{44}$ doit être évoquée. Saint Georges fut adopté comme figure protectrice par les Croisés en Terre sainte à l'époque médiévale.

Remarquons l'acuité du relief sur les conditions de la lente reconquête de ces régions par le catholicisme. Si les montagnes ont freiné l'avancée des Ottomans, elles ralentissent également l'emprise du catholicisme. Dans cette région de frontière, dans un diocèse encore trop peu structuré, les montagnes constituent un secteur assez mal maîtrisé. Au début du XVIII ${ }^{\mathrm{e}}$ siècle, l'islam y est encore si proche que certaines dévotions rassemblent chrétiens et musulmans. La crainte d'une confusion des genres n'est certainement pas absente lorsque l'évêque Martin Brajković (vers 1700) décrit l'affluence traditionnelle de catholiques, de « schismatiques » et de Turcs venus de loin pour vénérer saint Jean-Baptiste dans une église détruite au sommet du mont Velebit qui renfermerait la tête

42. Luigi Ferdinando De Marsigli, Relazioni dei confini della Croazia e della Transilvania a sua maesta cesarea (1699-1701) a cura di Raffaella Gherardi, Modena, 1986, Relazione vigesimasecunda, spedita dal campo di Globovatz, fra Novi e Castanovitz, ai 23 di maggio 1700, p. 355 (538r.) : Un marcatante di Segnia mando merci a Bagnalucca sulla fede della pace, e $i$ suoi uomini, fattisi turchi, s'usurparono i capitali. Egli stesso ando in persona al Capitano di Bagnalucca a chiedere giustizia, e li disse che se $i$ suoi uomini si erano fatti turchi, non per questo mutar dovea condizione la sua roba, e che percio ne domandava la restituzione.

43. Figure sculptée au sommet de l'autel latéral gauche de l'église paroissiale de Donji Kosinj.

44. Louis Réau, Iconographie de l'art chrétien, Paris, 1958, III/II, p. 572. 
du saint ${ }^{45}$. S'impose l'analogie avec le tombeau abrité dans la mosquée des Omeyyades à Damas, où les musulmans vénèrent la relique du chef de saint Jean-Baptiste. Les musulmans honorent habituellement le précurseur et prophète Jean-Baptiste, associé dans le message coranique à la personne de Jésus, dernier grand prophète avant Mahomet. En un lieu voisin, en 1700, au cour de la Lika, dans la petite bourgade de Lovinac, survient le miracle de l'apparition de saint Michel archange et de saint Jean-Baptiste ${ }^{46}$. Dans le récit, l'apparence de l'archange qui dissimule une moitié de son visage par un vêtement de couleur verte exprime-t-elle l'inachèvement d'une conversion ? La couleur de l'islam, émanation d'une tradition iconoclaste, couvre à demi l'image du messager de Dieu, autre référence à une sensibilité religieuse peut-être ambiguë : le culte de Michel archange est commun aux musulmans et aux chrétiens. Reprise sur les Turcs 11 ans auparavant, la bourgade de Lovinac est un poste fortifié frontalier encore proche de l'empire ottoman. De la même manière, la dévotion à saint Élie fortement ancrée dans le diocèse procède d'une ambivalence avec l'islam. Réputé avoir lutté contre les cultes des idoles, Élie est un prophète nommé llyass dans l'islam (et Ilija en croate), également vénéré par les musulmans qui croient que son retour précéderait le messie.

Pour surmonter ces doutes, le clergé catholique visite et administre jusqu'aux vallées les plus étroites, coincées dans les massifs

45. Lopašić, document CIV, p. 191 : ...In monte Velebich etiam nunc dirruta ecclesia S. Joannis bapt., olim et nunc miraculis clarissima, ad quam et catholici et schismatici et ipsi Turcae ex longenquis partibus devotionis gratia confluunt, votaque faciunt. In hac ecclesia dicitur fuisse caput s. Joannis baptistae...

46. Le père Marko Mesić, qui conduisit face aux Turcs la libération de la Lika et de la Krbava, envoie le 23 septembre 1700 depuis Mušaluk à l'évêque de Senj, Martin Brajković, la lettre dans laquelle il raconte qu'à Lovinac se produisent des choses étranges. «Le 12 septembre 1700 le commandant de Lovinac, Stanislav Kovačević, sa mère et son frère Paul ont eu dans leur maison les visions suivantes : devant eux se sont présentés deux hommes, l'un plus âgé portant la barbe, habillé en soutane noire de prêtre, et l'autre d'apparence plus jeune, portant sur ses épaules des vêtements verdâtres ; celui-là leur dit à l'occasion de la première apparition qu'ils avertissent le peuple afin qu'il célèbre mieux les fêtes de l'église et qu'il évite les gros travaux, qu'il respecte les prêtres et qu'il obéisse à toutes leurs prescriptions, qu'il construise les églises et prie avec ferveur (...). » 
montagneux. À la fin du $\mathrm{XVII}^{\mathrm{e}}$ siècle quelque 882 Turcs ont été baptisés en Lika, par les prêtres envoyés par l'évêque de Senj ${ }^{47}$. Quatre mosquées, à Perušić, Budak, Bilaj et Ribnik, furent transformées en églises catholiques dans lesquelles furent commencées la célébration de l'office et l'administration des sacrements ${ }^{48}$. Dans ce secteur, le réseau catholique du sacré est recomposé au cours des années 1690-1720. Les nouvelles églises font référence à l'obédience romaine, aux dogmes catholiques et à la monarchie autrichienne. Face aux orthodoxes ou face aux musulmans, les vocables sont emblématiques. Saint Pierre et saint Paul sont honorés dans l'église de bois à Ribnik. À Mušaluk, l'église est dédiée au Saint-Esprit. L'église de Perušić, au cœur d'une paroisse alors largement composée d'une population de musulmans convertis au catholicisme, est dédiée à la Crucifixion. L'affirmation catholique répond ainsi au Coran, selon lequel le Christ n'a pas été crucifié mais seulement substitué. Signe d'allégeance à l'empereur et à son fils, les églises de Bilaj et de Budak sont respectivement placées sous les vocables de saint Léopold et de saint Joseph. Enfin, jusqu'en 1779, l'archidiacre de Lika et Krbava sera fixé non loin de là, à (Lički) Novi ${ }^{49}$.

\section{UN DIOCÈSE CATHOLIQUE DE L'AUFKLÄRUNG}

Le territoire montagneux du diocèse de Senj-Modruš s'insère dans les marges impériales de l'Europe orientale où, durant les $\mathrm{XVIII}^{\mathrm{e}}$ et XIX ${ }^{\mathrm{e}}$ siècles, s'instaure une société nouvelle, sous l'impulsion de l'Aufklärung ${ }^{50}$. Tant par l'ancrage des clercs séculiers dans les paroisses isolées que par le rayonnement des missionnaires, l'Église catholique y imprime sa marque.

47. Lopašić, p. 28, document XXVII, I/. Un acte émanant du chapitre de la cathédrale de Senj, en date du 21 février 1696, signé du prêtre Wolfgang Vlatković, chanoine et chancelier du chapitre, en atteste.

48. Lopašić, p. 28, document XXVII. Autre acte du même auteur et de la même date.

49. Schematismus cleri dioecesium Segniensis et Modrusiensis, seu Corbaviensis pro anno 1879, Senj, p. 31.

50. Ruegg. 


\section{Le renforcement du réseau paroissial en montagne}

Pour mieux assurer le service religieux, une réorganisation des structures paroissiales est réalisée par les évêques successifs. En 1753 , le personnel religieux du diocèse est plus nombreux ; parmi les 274 prêtres séculiers, on dénombre 43 chanoines, 46 curés et 34 chapelains, auxquels s'ajoutent 54 franciscains, 31 pauliniens et 15 capucins. En tout, ce sont 374 clercs réguliers et séculiers qui desservent les 67117 fidèles du diocèse. Mais la répartition des clercs dans le diocèse est inégale. La ville de Senj, avec son chapitre cathédral de 12 chanoines et ses religieux franciscains et pauliniens, offre assurément un encadrement paroissial exceptionnel à ses quelque 2000 habitants. La situation des 2000 habitants d'Otočac et des 1500 habitants de Brinje est moins favorable avec deux clercs pour chacune des paroisses. Dans le diocèse de Modruš, l'encadrement des paroisses de Brod, Ogulin et Lešće semble inexistant. Il est bien faible à Gerovo (1 prêtre pour 1500 fidèles), à Moravice ( 1 curé pour 2000 fidèles), ou à Oštarije ( 1 curé pour plus de 1000 fidèles), au cœur de zones montagneuses. Néanmoins, certaines évolutions témoignent de l'accent porté sur les lieux retirés, tant par les clercs séculiers que par les clercs réguliers. Afin de remédier à la faible efficacité pastorale apparue à la périphérie des paroisses, notamment celles de grande étendue, l'impératrice Marie-Thérèse (1740-1780) décide, le 23 janvier 1755, que soient recueillies des informations sur le nombre des paroisses, succursales et églises des pays autrichiens. Le degré d'implication de l'évêque dans l'administration du diocèse est élevé : l'évêque Vuk Juraj Tcholić visite au moins à trois reprises son vaste diocèse montagneux (en 1748, 1753 et 1757). De nombreuses paroisses proches de la frontière turque sont enfin pourvues d'inventaires grâce à la générosité de l'impératrice Marie-Thérèse ${ }^{51}$. L'organisation diocésaine se renforce. L'évêque et les vicaires généraux, un pour chacun des

51. Mile Bogović, «Sadržaji izvješća ad limina senjske i modruške ili krbavske biskupije u Rimu od 1602. do 1919. godine », Senjski zbornik, 23, 1997, p. 182. 
deux diocèses de Senj et Modruš, sont secondés par des archiprêtres, mais également par des vicaires «extérieurs » (vicarii foranei) pour les régions intérieures du massif montagneux ${ }^{52}$, celui de transCapella (de l'autre côté des monts Kapela) dans le secteur d'Ogulin, et celui de Lika ${ }^{53}$. Loin de connaître le déclin noté par ailleurs pour leurs homologues dans le diocèse alpin de Brescia dans la république de Venise ${ }^{54}$, ces vicarii foranci conservent une dimension institutionnelle importante durant tout le $\mathrm{XVIII}^{\mathrm{e}}$ siècle. Leur fonction d'intermédiaire entre l'évêque et les curés leur donne une importance plus sensible dans les comtés de Lika et Krbava où le pouvoir épiscopal est dénigré par l'administration militaire des Confins. La juridiction diocésaine sur l'archidiaconé de Lika-Krbava fut provisoire jusqu'en 1752, lorsque la cour de Vienne la reconnut officiellement. Mais c'est seulement en 1833 que l'archidiaconé fut intégré pleinement dans les institutions pastorales du diocèse ${ }^{55}$.

La multiplication des paroisses et des chapellenies se confirme. Dans son rapport de 1733, l'évêque Benzoni propose que soient créées des chapellenies dans les paroisses de Smiljan, Budak, Pazarište et Perušić, et que Lovinac, succursale éloignée de la paroisse de Gračac, soit érigée en paroisse. Il indique que cette filiale est située au milieu des « schismatiques ${ }^{56}$. Des chapellenies indépendantes furent édifiées à Brušani relevant de la paroisse de Novi et à Bunić relevant de la paroisse de Čanak (Chanken) ${ }^{57}$. En 1753, l'évêque Vuk Juraj Tcholić note que trois nouveaux chapelains ont été installés à Blagaj, Brušane et Kaluđerovac ${ }^{58}$, toutes localités d'altitude. En 1767, l'évêque Pio Manzador octroie une aide financière au chapelain titulaire de l'église de Bruvno, fort éloignée de l'église mère de

52. Mentionnés en 1741 à l'époque de l'évêque Ivan Antoine de Benzoni, puis sous son successeur Vuk Juraj Tcholić.

53. Bogović, p. 180.

54. Luisa Andrighettoni, I vicariati foranei della valle Camonica nelle visite pastorali dal Concilio di Trento ad oggi, Brescia, 1976, p. 75 et ss.

55. Hoško, p. 269.

56. Hoško, note 18 p. 275 ; Cf. Status parochiarum, fo 55v : Filialis hace Ecclesia in Gracacz cum in medio Schismaticorum sit posita et 4 horis a Parochiali distans, necessarium est ut in Parochialem erigatur (...).

57. Hoško, p. 275.

58. Bogović, p. 180. 
Gračac ${ }^{59}$. Dans une contrée où vivent de nombreux orthodoxes, la présence d'un prêtre d'obédience romaine devrait désormais inciter les catholiques à rester dans leurs foyers ${ }^{60}$. L'évêque finance encore la construction de l'église de Notre-Dame-des-Douleurs à Zrmanja, autre succursale de la paroisse de Gračac, se trouvant à proximité du point où confinent les trois États vénitien, habsbourgeois et ottoman. En 1768, une commission visite toutes les paroisses de l'archidiaconé de Lika-Krbava afin d'estimer la situation des biens de ces paroisses et d'envisager la création de nouvelles stations pastorales, appelées « chapellenies indépendantes ${ }^{61}$ ». Les difficultés d'accès, notamment en hiver lorsque souffle la bura, et la nécessité de visiter, en été, les bergers dispersés dans les monts du Velebit ont motivé la fondation d'une chapellenie indépendante à Cesarica («Impériale »), filiale de la paroisse de Karlobag ${ }^{62}$. Cette chapellenie ne sera élevée au rang d'église paroissiale qu'en $1807^{63}$. Dans son rapport de visite ad limina de l'année 1773, l'évêque Pio Manzador fait le bilan de son épiscopat. La création de 11 chapellenies est indiquée ${ }^{64}$.

Un réseau de paroisses plus dense, dont les mailles enserrent davantage de localités, consolide le catholicisme. Cette évolution, sensible dans le secteur montagneux à l'Est de Senj pendant les dernières décennies du XVIIII siècle, a été étudiée par Darko Nekić65. L'évolution démographique conduit la localité de Senjska Draga, qui relevait jusqu'alors de la paroisse de Sveti Juraj, à bénéficier en 1723 de la construction de l'église abbatiale Sainte-Croix. En 1755, une paroisse y est fondée. Le ban paroissial de Senjska Draga est

59. Archives diocésaines de Senj, BAS, Protocollum commission mixtae seu conscriptio parochialium filiarumque ecclesiarum Archidiaconatus et districtus Liccano-Corbaviensis Dioecesis Modrussiensis sub jurisdictione inclyti Regiminis Licani existentium (1768), $\mathrm{f}^{\circ} 10 \mathrm{v}$.

60. Hoško, p. 273.

61. Hoško, p. 270.

62. Hoško, p. 274. Cf. Protocollum, $\mathrm{f}^{\circ} 16 \mathrm{r} \& \mathrm{v}$.

63. Schematismus cleri dioecesium Segniensis et Modrussiensis, seu Corbaviensis pro anno 1879, p. 29.

64. Bogović, p. 182.

65. Darko Nekić, «Senjska Draga - Kratka povijest mjesta i župe », Svjetionik, 1, 2000, p. 8-9. 
alors très étendu et y sont inclus plusieurs hameaux disséminés dans les environs. Une église avait été élevée au début du XVIII siècle à Majorija, à mi-chemin du col de Vratnik au sommet de la montagne, et quelques petites églises sont encore signalées à flanc de montagne. Mais l'abbé Homolić note : "Vu que les populations des villages avoisinants de Stubglia, Melnice et Stolac sont éloignées de cette église $[\ldots]$ de 4 à 6 heures de marche de l'abbaye Sainte-Croix, il conviendrait de faciliter leur incorporation à l'église paroissiale de Sainte-Croix selon les vœux du défunt Monseigneur Manzador ${ }^{66}$. » La décision fut prise conjointement par l'évêque et par l'administration militaire d'Otočac et actée le 6 novembre 1764 à Sveti Juraj. Les fidèles des alentours reçoivent les sacrements dans l'église de Sainte-Croix. En 1769, Stubalj et Melnice se séparent de la paroisse de Senjska Draga pour former une paroisse indépendante. La même année, l'église Saint-Michel est reconstruite à Vratnik ${ }^{67}$. Le village de Stolac, situé à mi-pente sur le versant maritime des monts Velebit, est donc rattaché sur décision du commandement militaire du généralat de Karlovac à l'administration paroissiale de Vratnik, localité au sommet du col dans les monts Velebit. Pourtant, l'abbé Vuk Homolić explique : "parce que ce peuple pendant l'hiver peut très difficilement aller dans le froid à Vratnik, je n'ai pas évité de leur faire avec ferveur et avec l'amour chrétien ce que le service de prêtre m'impose et j'ai assisté ce peuple dans ses besoins dans l'église Sainte-Croix parce que plus facile d'accès ${ }^{68} »$. Mais c'est l'ouverture en 1779 de la route Joséphine qui permet de mieux desservir les âmes. Dans le défilé où serpente la route de montagne, l'abbatiale Sainte-Croix est placée en un point de passage obligé : "Quand on a fait la route près de l'église, la citerne a été construite ici pour les besoins des voyageurs, le lieu est devenu de plus en plus connu, et l'église était quotidiennement visitée et ainsi la dévotion envers le crucifix a augmenté dans la proportion que dans cette église ont

66. Archives diocésaines de Senj, F II br. $7 \mathrm{~K}$ : manuscrit original écrit en italien après 1776 par Vuk Homolic, abbé de Sainte-Croix à Senjska Draga. Histoire de l'abbaye, de sa situation à cette date et des problèmes pastoraux.

67. Nekić, p. 9.

68. Archives diocésaines de Senj, F II br. 7 K. 
servi les prêtres réguliers et séculiers annuellement jusqu'à 181 messes et certaines années 190 et plus ${ }^{69}$. » La construction de la route est doublée d'une infrastructure permettant l'hébergement des voyageurs confrontés aux rigueurs du climat. L'abbé a souhaité ces aménagements : «pour l'accueil du peuple qui venait ici pour prier, et pour que le peuple des graničari (ou Grenzer) qui passait par ici puisse s'abriter dans cette maison, la nuit ou pendant les intempéries, et pour d'autres raisons comme il était prévu dans toutes les paroisses et vicariats dans ce généralat, il convient que ce généralat donne un ordre au capitaine de l'ingénieur De Sonath pour qu'il fasse le plan de la maison paroissiale ${ }^{70} »$.

Installé au cœur des montagnes, le prêtre s'intègre à un corps social isolé dont il est le cœur. L'activité pastorale y est durable ; un travail de fond est entrepris. Formé au collège Urbano de Rome, Giovanni Hreglianović, est de retour dans sa patrie en $1763^{71}$. Il est affecté par le vicaire général dans une petite paroisse au cœur des montagnes en qualité de chapelain du curé. À Kosinj, il exerce son ministère apostolique, écoutant les confessions, assistant les mourants et apprenant la pratique nécessaire à sa fonction. Il y termine un panégyrique du Très Saint Rosaire ${ }^{72}$. Le 17 juin 1764, il informe la Propaganda des œuvres qu'il conduit dans une chapellenie au milieu des populations "schismatiques", où il séjourne pendant cinq mois durant. Après avoir récupéré les «brebis malades », et le curé n'ayant plus besoin de ses services, il a regagné Senj pour assister le tribunal de pénitence et faire des prédications ${ }^{73}$. Le nouvel évêque Pio Manzador lui laisse espérer une cure d'âmes ${ }^{74}$. Le 29 septembre 1765, Giovanni Hreglianović est pourvu de la cure

69. Ibid.

70. Ibid.

71. SCPF, Série «Scritture riferite nei congressi » (SC) : Dalmazia 12, $f^{\circ}$ 315r. Lettre de Giovanni Hreglianovic en date de Senj le 7 août 1763.

72. SCPF, Série «Scritture riferite nei congressi » (SC) : Dalmazia 12, $\mathrm{f}^{\circ} 341 \mathrm{v}$.

73. Ibid., $\mathrm{f}^{\circ} 417 \mathrm{r}$ - 418r. Lettre de Giovanni Hreglianovic en date de Senj le 17 juin 1764.

74. SCPF, Série «Scritture riferite nei congressi » $(\mathrm{SC})$ : Ungheria e Transilvania 6, 1761-1777, $\mathrm{f}^{\circ} 145$. Lettre de Giovanni Hreglianovic en date de Senj le 25 juillet 1765. 
de Krmpote ${ }^{75}$. Mais, le 24 février 1766, il s'adresse à nouveau au cardinal préposé à la Congrégation de la Propaganda Fide pour faire avancer ses affaires. Il fait part de sa situation insatisfaisante ${ }^{76}$ et de la pauvreté de sa maison. Il souhaite obtenir la cure d'Oštarije, alors vacante ${ }^{77}$. Une grande disparité existe alors entre les paroisses de montagne les plus pauvres, comme Trsat, Saborsko, Fužine, Delnice, Podlapača, Čanak, Budak, Smiljan, Ribnik, dont les revenus dépassent rarement les 90 florins annuels, et les paroisses les plus peuplées et par conséquent les plus riches qui sont celles de Čabar (175 florins), de Slunj (196 florins) et d'Ogulin (200 florins), situées le plus souvent en Lika, comme Perušić (273 florins), Lovinac (230), Pazarište (203), Otočac (191) et Brinje ${ }^{78}$. Mais les ressources tirées du casuel ${ }^{79}$ ne traduisent pas la réalité des revenus du curé soumis au régime de la portion congrue. Carriérisme et souci matériel peuvent donc provoquer l'instabilité du clerc séculier. Cependant, l'action d'un modeste pasteur d'âmes peut être complétée par les formes d'apostolat plus spectaculaires adoptées par les missionnaires de passage.

\section{Les missions de montagne}

Même dans l'arrière-pays, traversé par les troupeaux ovins en transhumance, ponctué de fertiles poljés alternant avec des entablements rocheux, milieu idéal pour l'élevage des caprins, les bergers vivent une partie de l'année dans leur stanište, sorte d'étable isolée. Les missionnaires s'attachent à visiter ces chrétiens dispersés et confinés dans leurs montagnes. Ainsi le père franciscain Giacinto Alemagna,

75. SCPF, Série « Scritture riferite nei congressi » (SC) : Dalmazia 12, $\mathrm{f}^{\circ}$ 570r. Lettre de Giovanni Hreglianovic en date de Senj le 29 septembre 1765.

76. Ibid., f $\mathrm{f}^{\circ} 632 \mathrm{r}-\mathrm{v}$. Lettre de Giovanni Hreglianovic en date de Krmpote le 24 février 1766.

77. Ibid., $\mathrm{f}^{\circ} 632 \mathrm{v}$.

78. Voir sur les ressources des clercs séculiers J. Burić, Le diocesi di Segna e di Modrussa durante l'episcopato di Giovanni Antonio Benzoni, 1730-1745, Roma, 1968, p. 45.

79. E. Sladović, Povesti biskupijah senjske i modruške ili krbavske, Trst 1856, p. 293. 
missionnaire apostolique en Croatie et Carniole, œuvre dans le massif de Modruš où, en 1729, il ramène au catholicisme plusieurs centaines de personnes, parfois par localité entière. Le religieux a institué des confréries, édifié des hospices, églises et calvaires, entre autres œuvres pies dont il a gratifié cette région ${ }^{80}$. Le réseau du sacré est ainsi reconstruit. Dans une lettre en date d'Ogulin le 24 février $1730^{81}$, adressée à la Propaganda, le père Alemagna note le caractère barbare des âmes de ces régions, dans l'un et l'autre diocèse (Senj, Modruš et Zagreb ?) ${ }^{82}$. Il fait par exemple état de superstitions idolâtres dans un village de 35 maisons morlaques $^{83}$. Le clergé des confins estime l'œuvre du père Alemagna, déjà connu dans le diocèse où il a prêché la parole de Dieu pendant quatre ans ${ }^{84}$. Le missionnaire est réputé avoir fait des prodiges parmi les infidèles ${ }^{85}$, avoir extirpé le vice, éradiqué les réunions dangereuses et répandu les vertus, ramené plusieurs « schismatiques » vers l'Église (catholique), apaisé les querelles ${ }^{86}$. Toutefois, les débordements d'une population attachée à la tradition de la vengeance personnelle préoccupent le clergé catholique ${ }^{87}$. En 1747, ce type de tensions entre clans rivaux et parfois entre membres d'une même famille, cousins et parents de degré divers, reste d'actualité et les missionnaires jésuites tentent d'y remédier ${ }^{88}$.

80. SCPF, Série « Scritture riferite nei congressi » (SC) : Ungaria/Transilvania 5 (1728-1760), f ${ }^{\circ}$ 41r-v. Voir également SCPF, Scrit. Rif. Vol. 666 (année 1729), f ${ }^{\circ}$ 77-78. Relazione di tutto l'operato del Molto Reverendo P. Giacinto Alemagna Missionario Apostolico in Croazia e Cragnio del 1729 fedelmente data dalli Giovani all'illustrissimo et Rev.mo Monsignore Vescovo di Segna, Modrussa e Corbavia.

81. Ibid., f ${ }^{\circ} 45 \mathrm{r}-\mathrm{v}, 48 \mathrm{r}-\mathrm{v}$ : lettre du père Alemagna en date d'Ogulin, le 24 février 1730.

82. Ibid., f $\mathrm{f}^{\circ} 4 \mathrm{r}$.

83. Ibid., fo $45 \mathrm{r}$.

84. Ibid., f ${ }^{\circ} 49$ r. Lettre en date d'Ogulin le 4 avril 1730, adressée à la Propaganda par plusieurs clercs séculiers, curés et chapelains des confins militaires.

85. Ibid., $\mathrm{f}^{\circ} 49 \mathrm{v}$.

86. Ibid., $\mathrm{f}^{\circ} 49 \mathrm{r}$.

87. Cet aspect des mentalités locales a été mis en évidence par Josip Burić dans sa thèse consacrée à l'épiscopat de Jean Antoine de Benzoni de 1730 à 1745.

88. ARSI, Austr. 204, $\mathrm{f}^{\circ}$ 52r. 
À la limite septentrionale du diocèse de Modruš, « contiguë à la Carniole », les jésuites visitent Gerovo en 1747. Le caractère inhospitalier du lieu est souligné : les missionnaires ont du mérite à parvenir au sommet inculte et neigeux des montagnes ${ }^{89}$. Dans ces régions, l'audience des jésuites n'est pas négligeable : à Čabar, ce sont jusqu'à 1700 personnes qui participent à la communion (festin sacré). Dans le Gorski Kotar, à Fužine, les missionnaires donnent la communion à mille personnes ${ }^{90}$. Les missionnaires ouvrent des stations en contrebas des montagnes, en bordure de la mer Adriatique. Ainsi en 1748, la mission Dalmatica fait halte dans les bourgades portuaires de Sveti Juraj et de Jablanac ${ }^{91}$. La hiérarchie diocésaine a encore recours aux missions jésuites au cours de la seconde moitié du $\mathrm{XVIII}^{\mathrm{e}}$ siècle. De nouvelles stations en pays montagneux sont mentionnées, ainsi en 1767 Sinac et Kuterovo. À la fin du siècle, dans les pays germaniques et plus précisément dans la province jésuite d'Autriche, les missions s'adressent davantage aux paroissiens des villages et hameaux dispersés ou plus reculés dans les forêts ${ }^{92}$.

Lors de la mission des jésuites en 1767 dans le doyenné de Otočac, si un millier d'âmes se rassemble en présence des missionnaires, des populations restent dispersées sur de larges étendues de territoire. Il est impossible de réunir tous ces chrétiens en une seule station. Les jésuites expliquent ainsi le choix de stations complémentaires, comme Sinac et Lešće ${ }^{93}$. Dans ces lieux, les populations affluent, «impatientes d'être instruites de la pénitence ». Les jésuites y accomplissent une cérémonie liturgique avec les grandes laudes et la déclamation des hymnes ambrosiens ${ }^{94}$. La mission se poursuit à Kosinj, station qui rassemble des populations catholique

89. Ibid., f $\mathrm{f}^{\circ} 52 \mathrm{v}$.

90. Ibid., $\mathrm{f}^{\circ} 53 \mathrm{r}$.

91. ARSI, Austr. 205, fo $24 \mathrm{r}$.

92. Louis Châtellier, «À propos de la déchristianisation au XVIII ${ }^{\mathrm{e}}$ siècle », in La vie, la mort, la foi, le temps. Mélanges offerts à Pierre Chaunu, textes réunis par Jean-Pierre Bardet et Madeleine Foisil, 1993, p. 487 et note 8 p. 487.

93. ARSI, Austr. 222, fo $21 \mathrm{v}$.

94. Ibid., f $\mathrm{f}^{\circ} 22 \mathrm{r}$. 
et «schismatique». Dans cette paroisse, quelque 1500 personnes affluent auprès des missionnaires ${ }^{95}$. La mission a été soutenue par des personnalités laïques ou ecclésiastiques, comme François de Paule Fisko, vice-commandant à Otočac, le distingué et noble seigneur Kollaković, le révérend père et chanoine Wolfgang de Homolić, l'archiprêtre Joseph Zottoni ${ }^{96}$. Les jésuites ont néanmoins rencontré des limites dans leur apostolat. Ils n'ont pu répondre aux besoins des habitants de la Lika, région dont l'accès leur est impossible puisque les religieux capucins de Karlobag y disposent du monopole missionnaire ${ }^{97}$. Dans sa communication du 17 janvier 1769, le provincial des capucins de Styrie précise qu'à Kaniža, en Krbava et à Karlobag, deux capucins mènent les missions apostoliques pendant trois mois entiers, avec des résultats évidents et que le nombre des communiants dépasse les 20000 . Bien que les «schismatiques» ou d'autre part les « bandits » assistent plus difficilement aux missions, plusieurs sont ramenés à la foi catholique. Cette tâche de mission apostolique reste exposée à différents dangers, mais malgré tous les inconvénients les capucins poursuivent leur travail ${ }^{98}$.

Au XVIII ${ }^{\mathrm{e}}$ siècle, d'un simple glacis militaire, le diocèse montagneux de Senj-Modruš est devenu une frontière intérieure. Les prêtres séculiers renforcent leur emprise sur la vie quotidienne, tandis que les missionnaires couvrent des espaces plus étendus en répétant leur pérégrination dans les lieux les plus isolés. Une plus grande efficience de l'administration diocésaine, adaptée aux exigences de la géographie physique et humaine, a permis d'encadrer l'ensemble des catholiques. La modernité voulue par la maison d'Autriche ne réduit cependant pas l'originalité de la société des confins.

\section{Luc Oreskovic}

c/o Société R. J. Boskovic (Recherche sur l'aire culturelle croate) Université Paris IV - Sorbonne, 1 rue Victor Cousin, 75005 Paris

95. ARSI, Austr. 222, $\mathrm{f}^{\circ} 22 \mathrm{r}$.

96. Ibid., f $22 \mathrm{v}$.

97. Ibid.

98. Nikola Bašnec, «Dolazak kapucina i njihova misijska djelatnost $\mathrm{u}$ Lici i Krbavi nakon oslobođenja od Turaka 1689. », Riječki teološki časopis, 2, 1999, p. 289. 
